\title{
A NEW VIEW OF THE SUPERSOFT X-RAY SOURCE CAL 87 OBSERVED WITH XMM-NEWTON
}

\author{
M. Orio, ${ }^{1,2}$ K. Ebisawa, ${ }^{3}$ J. Heise,${ }^{4}$ and W. Hartmann ${ }^{4}$
}

Cal 87 was observed with with $X M M$ Newton in April of 2003. The source shows a rich emission spectrum, where lines can be identified if they are red-shifted by $700-1200$ $\mathrm{km} \mathrm{s}^{-1}$. These lines seem to have been emitted in a wind from the system. The eclipse is observed to be shifted in phase by $0.03 \phi_{\text {orb }}$, where $\phi_{\text {orb }}$ is the phase of the optical light curve.

\section{THE OBSERVED X-RAY SPECTRUM}

Cal 87 was observed with XMM Newton on April 18-19 2003, for 21.8 hours (two full orbital cycles). The background corrected count rate measured in the two RGS-1 and RGS-2 instruments in the 0.33$2.5 \AA$ range is $0.0764 \pm 0.0012$ and $0.0653 \pm 0.0011 \mathrm{cts}$ $\mathrm{s}^{-1}$, respectively. The spectrum clearly appears to be an emission line one. There is an obvious similarity with the $\mathrm{X}$-ray grating spectra of this source, (Motch et al. 2002, Bearda et al. 2002), however Cal 87 displays a much softer spectrum. Tentative line identification for the emission features in the RGS spectra indicates a red shift of several identifiable lines with velocities in the $700-1200 \mathrm{~km} \mathrm{~s}^{-1}$ range. We conclude that these lines must be originated in a wind. The EPIC spectra, despite pile-up effects that cannot be completely corrected for, offer a broader energy range $(0.2-10 \mathrm{keV})$, and allow us to conclude that the central white dwarf is not visible at any time, even outside of eclipse.

\section{THE LIGHT CURVE}

The eclipse already observed in X-rays is observed again with XMM-Newton. It is more definite and deeper in the EPIC in the RGS light curve, not only because of the better $\mathrm{S} / \mathrm{N}$, but also because the depth of the eclipse is greater at lower energy.

\footnotetext{
${ }^{1}$ INAF - Turin Astronomical Observatory, Italy.

${ }^{2}$ Department of Astronomy, $U$ Wisconsin at Madison, USA.

${ }^{3}$ INTEGRAL Science Data Centre, Versoix, Switzerland

${ }^{4}$ SRON National Institute for Space Research, Utrecht, The Netherlands.
}

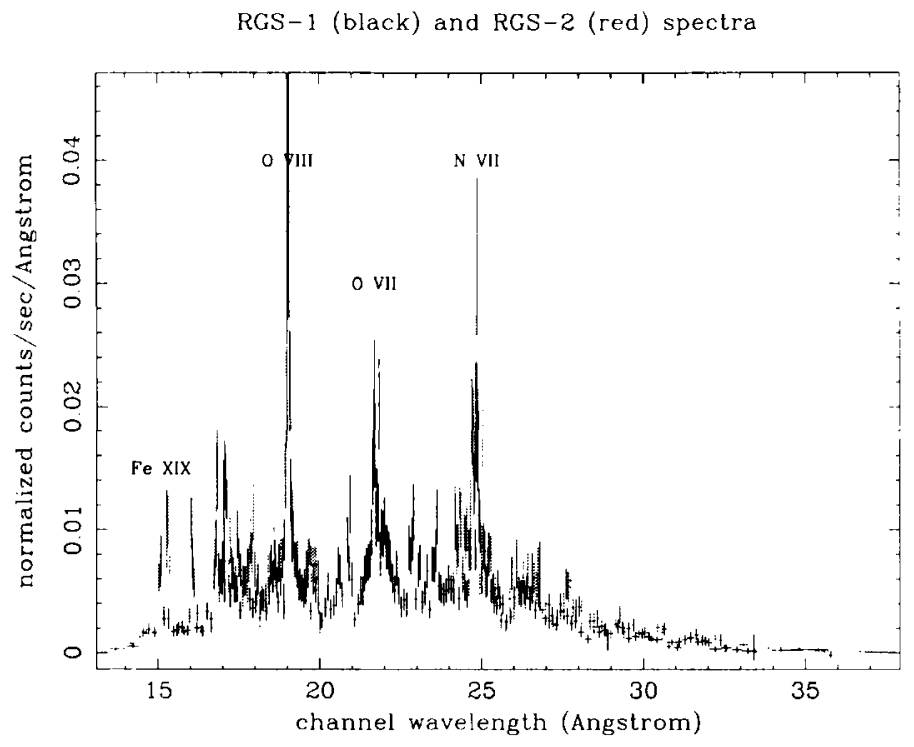

Fig. 1. The spectrum observed with the two RGS gratings. The RGS-1 spectrum is shown in black, the RGS-2 spectrum is in red. Some line identifications are labelled. No significant signal above the background is detected at wavelengths below $15 \AA$.

It is shifted by $\Delta \phi_{\text {orb }}=0.03$ with respect to the eclipse observed at optical wavelengths. We speculate that this may be so because the X-rays are emitted by the Accretion Disk Corona, while the optical radiation originates instead from the disk.

\section{REFERENCES}

Bearda, H., et al. 2002, A\&A, 385, 511

Motch, C., Bearda H., and Neiner C. 2002, 385, 91 